\title{
Search for Evidence of a Clock Related to the Solar 154 Day Complex of Periodicities
}

\author{
P. A. Sturrock and T. Bai \\ Center for Space Science and Astrophysics \\ Stanford University, Stanford, CA 94305-4055, U.S.A.
}

Accepted 1992 March 17

To appear in The Astrophysical Journal 1992 September 10 


\title{
SEARCH FOR EVIDENCE OF A CLOCK RELATED TO THE SOLAR 154 DAY
}

\section{COMPLEX OF PERIODICITIES}

\author{
by P.A. Sturrock and T. Bai \\ Center for Space Science and Astrophysics, \\ Stanford University, Stanford, CA 94305
}

\begin{abstract}
Evidence that has recently been compiled (Bai and Sturrock 1991) indicates that the enigmatic 154-day periodicity in solar activity may be viewed as part of a complex of periodicities that are approximate multiples of 25.8 days, suggesting that the Sun contains a "clock" with frequency in the range $440-463 \mathrm{nHz}$. The clock may comprise either an oscillator or a rotator, each of which may be either real or virtual.

We have reconsidered a previous spectrum analysis of the Zurich sunspotnumber sequence by Knight, Schatten and Sturrock (1979) which revealed a sharp, persistent and significant periodicity with a period of 12.072 days, corresponding to a frequency of about $958.8 \mathrm{nHz}$. This periodicity may be regarded as the (second) upper sideband of the second harmonic $\left(2 v_{R}+2 v_{E}\right)$ of a fundamental frequency of 447.7 $\mathrm{nHz}$ that is clearly within the search band. In this expression, $v_{\mathrm{R}}$ is the sidereal frequency of the hypothetical rotator and $v_{E}$ is the frequency $(31.69 \mathrm{nHz})$ of the Earth in its orbital motion around the Sun. In analyzing sunspot area data derived from the Greenwich data set, and on noting that any frequency is defined only to within the Nyquist frequency, we find clear evidence not only for the upper sideband of the second harmonic, but also for the second harmonic $\left(2 v_{R}\right)$ and the lower sideband of the second harmonic $\left(2 v_{R}-2 v_{E}\right)$. There is no strong peak at the fundamental frequency in the Greenwich data, but there is in the Zurich sunspot data.
\end{abstract}


The effect of a linear oscillator is, to lowest order in the amplitude, the same as the combined effect of two rotators of opposite polarities. A rotator that has arbitrary orientation with respect to the ecliptic may influence the outer layers of the Sun and thereby modulate the occurrence of solar activity such as sunspots. By analyzing a simple model, we find that such a rotator would influence surface activity in such a way that the spectrum of a "signal" (such as the record of sunspots), as seen from Earth, would contain components with frequencies that are certain integral combinations of $v_{R}$ and $v_{E}$. The amplitudes of the various components depend sensitively on $\Theta$, the angle between the axis of the rotator and the axis of the Earth's orbital motion. This simple model therefore offers a kinematical (but not a dynamical) interpretation of the sunspot spectrum.

The present analysis, while offering support of our conjecture that the Sun contains a clock that regulates the 154-day complex of periodicities, cannot distinguish between an oscillator or a rotator (that might be a traveling wave), nor between a real rotator or a virtual rotator (that might be an apparent traveling wave due to the aliasing effect of an oscillator in a rotating system). Further analysis of sunspot and other data sets will be required to confirm the existence of such a clock and (if it is real) to determine its physical nature. 


\section{INTRODUCTION}

Solar activity exhibits many periodicities and quasi-periodicities, most prominently periodicities near 27 days related to solar rotation, and periodicities near 11 years and 22 years related to the solar cycle (Schwabe 1844; Hale 1924). In recent years, evidence has accumulated supporting a periodicity at about 154 days (Rieger et al. 1984; Ichimoto et al. 1985; Bai and Sturrock 1987; Bai and Cliver 1990), and another at about 2.2 years (Sakurai 1979; Haubold and Gerth 1990), quite close to the quasi-biennial oscillation that is known to occur in the Earth's upper atmosphere (see, for instance, Andrews, Holton and Leovy 1987). At this time, there is no accepted theory for either of these two enigmatic periodicities.

In this paper we address the 154 -day periodicity. This periodicity was discovered by analyzing the occurrence rate of gamma-ray flares detected by the gamma-ray spectrometer aboard the Solar Maximum Mission (SMM) for the interval 1980 - 1983 (Rieger et al. 1984). After this discovery, many researchers confirmed the existence of this periodicity in solar cycle 21 (1976 - 1986) by analyzing various indicators of solar activity (see Bai and Cliver 1990 and references therein). This periodicity was also found in some activity indicators of solar cycles 19 and 20 (see references in Bai and Cliver 1990). It is also significant that the power spectrum of solar diameter measurements made during the interval 1683 - 1718 exhibits a 6 sigma peak at 155 days (Ribes et al. 1989). This evidence has led to the general - but not universal - conclusion that the 154-day periodicity represents a fundamental property of the Sun.

The most obvious interpretation to be considered is the hypothesis that, since the rotation rate of the solar envelope varies with latitude and radius, different regions of the Sun with slightly different rotation rates meet every 154 days and interact to enhance flare activity. We have tested this conjecture by analyzing the 
solar coordinates of the major flares observed by the Hard X-ray Burst Spectrometer (HXRBS) aboard SMM and found that this evidence does not support this proposal (Bai and Sturrock 1987).

An alternative idea, advanced by Wolff (1983), is similar to the preceding proposal except that one considers the interaction of active bands that are due to $g$ mode oscillations in the radiative core of the Sun (an example of what we refer to as "virtual rotators"). According to Wolff (1983), the $75 \mathrm{nHz}$ (155-day) periodicity is due to the interaction of active bands of $l=2$ g-mode oscillations and active bands of $l=3 \mathrm{~g}$ mode oscillations. In this picture, flares produced during the peak phases of the 154day periodicity are expected to be concentrated in the active bands associated with each oscillation mode. By noting the rotation rates of these oscillations as they were given by Wolff (1983), we were able to test his proposal by examining the distribution of flares in these rotating coordinate systems (Bai and Sturrock 1987). Once again, we found that the evidence does not support the proposal.

Until recently, we could see no other possible interpretation of the 154-day periodicity. However, recent investigations have shown that this periodicity may be viewed as simply one member of a complex of periodicities. In 1987, we found a 51day periodicity for cycle 19 (1955 - 1965) by analyzing the occurrence rate of major flares selected on the basis of the comprehensive flare indices (CFI) (Bai 1987). Since the CFI compilation of Dodson and Hedeman $(1971,1976,1981)$ covers the time interval 1955 - 1979, we undertook to analyze the occurrence rate of CFI major flares since 1966. This analysis yielded significant peaks at 84, 104, and 129 days (Bai and Sturrock 1991). The 84-day peak is the strongest in the spectrum formed from the interval 1966-76, but the 129-day peak is the strongest in the spectrum formed from the interval 1966-1979. In the power spectrum of the occurrence rate of flares selected by peak microwave emission for cycle 20 , the strongest peak is found at 78 days (Bogart and Bai 1985). In the power spectrum of the daily sunspot areas for the 
interval 1971-78, the strongest peak is found at 78 days (Bai and Sturrock 1991). Of the above-mentioned periods, all but 84 days are close to integral multiples of 25.8 days. We estimate that the probability that four out of the five peaks $(51,78,84,102$, and 129 days) fall by chance near integral multiples of 25.8 days is $3 \times 10^{-5}$ (Bai and Sturrock 1991).

For these reasons, we have been led to propose that there is a "clock" inside the Sun with a fundamental period of about 25.8 days, and that this clock somehow excites subharmonics in the outer layers of the Sun (Bai and Sturrock 1991). The "clock" could in principle comprise any periodic process in the solar interior. It could be due to the rotation of some part of the Sun, that we refer to as a "real rotator," or it could be due to a rotating-wave disturbance (such as was considered by Wolff (1983)) that we refer to as a "virtual rotator." It is also possible that the clock is due to a dipole-type or quadrupole-type oscillation, or something more complicated. However, to lowest order in the amplitude of the disturbance, we may represent such an oscillator by a combination of rotating-wave patterns, i.e. by a combination of virtual rotators. For this reason, it is sufficient for our present purpose to consider only the problem of detecting the existence of a real or virtual rotator within the Sun. The fact that the "154 day" periodicity varies in period over the range 150-158 days yields a search band of $440-463 \mathrm{nHz}$ for this fundamental period.

To search for evidence of this hypothetical clock, we have Fourier-analyzed the daily Greenwich sunspot areas and daily Zurich sunspot numbers. It is to be expected that, if the clock has an intrinsic frequency $v_{R}$, an observer on Earth might detect not only this frequency and its harmonics, but also "alias" frequencies or "sidebands" that are shifted by multiples of $v_{E}$, the frequency of the Earth's orbital motion around the Sun. The results of the spectrum analysis are discussed in $\S 2$. The spectrum of the Greenwich area data shows no notable peak in the search band for the fundamental, but it exhibits a very strong peak at $894.3 \mathrm{nHz}$ (period 12.94 days) 
that may be interpreted as the harmonic of the fundamental frequency. Furthermore, there are also a very strong peak at $958.8 \mathrm{nHz}$ (period 12.07 days) and a strong peak at $830.6 \mathrm{nHz}$ (period 13.93 days). These two peaks are separated from the $894.3 \mathrm{nHz}$ peak by about two times the Earth's orbital frequency. It appears, therefore, that the frequencies $2\left(v_{R}-v_{E}\right), 2 v_{R}$ and $2\left(v_{R}+v_{E}\right)$ are all evident in the Greenwich data. In reviewing the spectrum of the Zurich data, we found a strong peak at $447.9 \mathrm{nHz}$ (25.84 days), that may clearly be identified with the fundamental frequency. The peak at $958.8 \mathrm{nHz}$ is quite conspicuous in the Zurich data, as was noted some time ago by Knight, Schatten, and Sturrock (1979).]

In $\S \S 3$ through 6 , we investigate how an obliquely rotating pattern would be perceived by an Earth-based observer, and we find that it is indeed reasonable that such a pattern would produce peaks at $2\left(v_{R}-v_{E}\right), 2 v_{R}$ and $2\left(v_{R}+v_{E}\right)$. In $\S 3$, we introduce a sequence of coordinate systems, each step of which involves only a simple rotation about a common axis. In $\S 4$, we introduce a "distortion function," that we assume to be time invariant in an obliquely rotating coordinate system, and represent it as a sum of spherical harmonics of up to second order. By applying successive coordinate transformations, we find the coefficients of the spherical harmonics that describe the distortion with respect to an ecliptic-based inertial coordinate system. The coefficients of these spherical harmonics vary with frequencies that are multiples of $v_{R}$. In $\$ 5$, we express the "acceptance function" (or "visibility function") for the Earth-based observer as a sum of spherical harmonics of up to second order. In the ecliptic-based inertial coordinate system, the coefficients of the spherical harmonics that represent the acceptance function change with frequencies that are multiples of $v_{E}$, due to the Earth's revolution around the Sun. In $\S 6$, we show that the signal detected by an Earth-based observer has a number of components, among them components varying with frequencies of $V_{R},\left(V_{R}-v_{E}\right),\left(V_{R}\right.$ $\left.+v_{E}\right),\left(v_{R}-2 v_{E}\right),\left(v_{R}+2 v_{E}\right), 2 v_{R}, 2\left(v_{R}-v_{E}\right)$ and $2\left(v_{R}+v_{E}\right)$, and interpret the three 
frequencies, 830.6, 894.3, and $958.8 \mathrm{nHz}$ of prominent peaks in the power spectrum of the Greenwich data as $2\left(v_{R}-v_{E}\right), 2 v_{R}$ and $2\left(v_{R}+v_{E}\right)$.

Possible implications of these results are discussed further in $\S 7$. The research presented in this article describes the first stage of an ongoing investigation. The results of research now in progress, that will hopefully clarify some of the questions left hanging in this article, will be presented in due course.

\section{DATA ANALYSIS}

The longest sequence of daily measurements of solar activity is the Zurich sunspot number tabulation. Knight, Schatten and Sturrock (1979) investigated the spectrum of Zurich daily sunspot numbers over the interval 1849 January 7 to 1970 November 28, searching for evidence of a rapid rotator with a period of order 12 days that had been suggested by Dicke (1976). They found a strong peak that appeared to be statistically quite significant; this peak has a period of 12.072 days corresponding to a frequency of $958.8 \mathrm{nHz}$, that we here refer to as the "KSS periodicity." At that time, Knight et al. conjectured that this frequency might be the "synodic" rotation frequency of the core of the Sun or the second harmonic of that frequency. We decided to investigate the possibility that the KSS periodicity may be related to a hypothetical clock that regulates the "154-day" complex of periodicities.

These periodicities vary somewhat in period from one sunspot cycle to another. The "154-day" periodicity (the strongest and best defined of this complex of periodicities) varies in period over the range 150-158 days. Since we identify this with the sixth sub-harmonic, this corresponds to a search band of $440-463 \mathrm{nHz}$ for the fundamental period. The search band for the second harmonic $\left(2 v_{R}\right)$ is therefore $880-926 \mathrm{nHz}$, that of the lower sideband $\left(2 v_{R}-2 v_{E}\right)$ is $817-863 \mathrm{nHz}$, and that of the upper sideband $\left(2 v_{R}+2 v_{E}\right)$ is $943-989 \mathrm{nHz}$. If the KSS periodicity is due to an oblique rotator, it can be identified only with the upper sideband of the second harmonic. If 
we tentatively make this identification, we then infer that the fundamental period is close to $447.7 \mathrm{nHz}$.

We have examined the sunspot spectrum developed by Knight et al. (1979), and we find that there is indeed a peak near $447.7 \mathrm{nHz}$. We find a stronger peak at $447.9 \mathrm{nHz}$ (indeed, this is the peak with the largest power [before normalization] within the entire period range $\mathrm{P}<27$ days), and a neighboring peak at $447.3 \mathrm{nHz}$ (see Fig. 1). When we examine the spectra of the first half and second half of the total interval, we find peaks at $447.7 \mathrm{nHz}$ and $448.2 \mathrm{nHz}$, respectively. Even if the Sun does in fact contain a clock with a well defined frequency that modulates solar activity, the natural variation of solar activity will modify the spectrum so that the strength and location of the peak will vary in time (Scherrer 1983). If the Sun contains a virtual rotator, for instance the excitation of a traveling-wave instability, the frequency might vary considerably as a function of time. Considering once more the entire data run, the normalized power of this peak is 5.3. The probability of finding a peak of this power in the $447-448.5 \mathrm{nHz}$ interval by chance is

$$
P_{1}=6 \exp (-5.3)=0.03
$$

where 6 is the number of independent frequencies. This step in the investigation therefore offers some evidence in support of the hypothesis that there is an oblique rotator within the Sun, with a sidereal rotation frequency in the range 447-448.5 $\mathrm{nHz}$, that is related both to the 154-day complex of periodicities and to the KSS periodicity.

We have also examined another related set of data, namely the record of sunspot activity compiled by the Greenwich Observatory. We have been provided with a record of the total sunspot area, derived from the more complete Greenwich data compilation, over the interval 1879 January 1 to 1982 August 15 . We have 
accordingly carried out a spectrum analysis of these data, and the results are shown in Figure 2. In examining the interval $447-448.5 \mathrm{nHz}$, we find once more that there is a peak at $447.9 \mathrm{nHz}$, with a secondary peak at $447.2 \mathrm{nHz}$ (Fig. 2d). These peaks are close to those found in the Zurich sunspot number spectrum, but not as striking. We searched for sidebands of the fundamental period, but these are either weak or absent.

It is clear that, if our interpretation of the KSS periodicity (and the periodicity near $448 \mathrm{nHz}$ ) is correct, we may find peaks in the spectrum also at the frequencies $2 v_{R}-2 v_{E}$ and $2 v_{R}$. If we regard the fundamental as located in the range 447-448.5 $\mathrm{nHz}$, we are led to search the bands 830.6 to $833.6 \mathrm{nHz}$ and 894 to $897 \mathrm{nHz}$. We find a very strong peak at $894.3 \mathrm{nHz}$ with normalized power 12.4 (Fig 2b). The probability of finding this peak by chance is given by

$$
P_{2}=12 \exp (-12.4)=4.9 \times 10^{-5}
$$

where the factor 12 is the number of independent frequencies in the 894 to $897 \mathrm{nHz}$ interval. We also find a peak at $830.6 \mathrm{nHz}$, with normalized power 4.3 (Fig. 2c). The probability of finding this peak in the $830.6-833.6 \mathrm{nHz}$ range by chance is

$$
P_{3}=12 \exp (-4.3)=0.16
$$

Hence the joint probability of finding these two peaks in the two search intervals is about $8 \times 10^{-6}$.

The above argument depends upon the previous analysis by Knight et al. (1979). We have decided also to analyze the available data without invoking this previous analysis. We chose to search the spectrum over the entire band $817 \mathrm{nHz}$ to $989 \mathrm{nHz}$, encompassing the harmonic and both sideband regions corresponding to the "154 day" period being located in the range $150-158$ days; the width of this band 
is $172 \mathrm{nHz}$, corresponding to about 57 bins each of width $3 \mathrm{nHz}$. Of the four largest peaks, we find that three form a sequence with step size 21 bins, or $63 \mathrm{nHz}$, that is the nearest integral approximation to $2 v_{E}$. Given a sequence of $n$ bins, the probability that three bins, selected at random, will form a sequence with two separations each of $q$ bins is

$$
P_{4}=(3 !) \cdot([n-2 q] / n) \cdot(1 /[n-1]) \cdot(1 /[n-2])
$$

Hence, given four bins (corresponding to the four largest peaks), the chance that three of the bins will form a sequence with two equal separations of $q$ bins each is

$$
P_{5}=4 .(3 !) \cdot([n-2 q] / n) \cdot(1 /[n-1]) \cdot(1 /[n-2]) .
$$

For $\mathrm{n}=57$ and $\mathrm{q}=21$, this yields $\mathrm{p}=0.002$. That is to say, there is a probability of only $0.2 \%$ of finding such a sequence by chance among the four largest peaks. Note that this estimate involves only the Greenwich data. It takes no account of the fact that the Zurich spectrum shows a strong peak at exactly the frequency corresponding to the three frequencies selected in the Greenwich spectrum. Furthermore, this estimate takes no account of the impressive strength of two of the three peaks: the peak at $958.8 \mathrm{nHz}$ (the KSS periodicity) has normalized power $\mathrm{S}=11$, so that the probability that this peak occurs by chance in a bin of width $3 \mathrm{nHz}$ is $10 \exp (-11)$, i.e. 2 $10^{-4}$. Noting that there are $n-2 q$, i.e. 15 , combinations of three bins separated by $2 v_{E}$, the chance that peaks of the given strengths would be found by chance is seen to be $15 \times 0.14 \times 610^{-5} \times 210^{-4}$, i.e. less than $3 \times 10^{-8}$.

There appears to be non-negligible evidence that the Sun does indeed contain some form of clock that regulates the 154-day complex of periodicities. In the following sections, we develop and analyze a very simple model for an oblique rotator that may be representative of such a clock. 


\section{COORDINATE SYSTEMS}

We adopt rectangular coordinates $(x, y, z)$ that we denote also in vector notation as $x$. It is convenient to introduce the following five coordinate systems with the common origin at the center of the Sun:

r-system: the $\mathbf{x}_{\mathbf{r}}$ coordinate system rotates with the rotator, and is aligned in such a way that the rotation axis of the rotator is parallel to the $\mathrm{z}$ axis.

f-system: the $x_{f}$ coordinate system is fixed in space, and is also aligned so that the rotation axis of the rotator is parallel to the $\mathrm{z}$ axis.

e-system: the $x_{e}$ coordinate system is fixed in space and is aligned in such a way that the ecliptic lies in the $x-y$ plane.

$t$-system: the $x_{t}$ coordinate system has its $z$-axis fixed in the direction of the axis of the ecliptic, but rotates with the Earth in such a way that the Earth always lies on the $x$-axis.

s-system: the $x_{s}$ coordinate system is such that the $z$-axis always points to the Earth, and the $x$-axis is antiparallel to the axis of the ecliptic.

We now examine the relationship between the above systems of coordinates. The "ecliptic" (e) coordinate system is such that that Earth's orbit lies in the $x-y$ plane and rotates in a positive sense with respect to the $z$-axis. We adjust the $x-y$ axis so that the axis of rotation of the rotator lies in the $x_{e}-z_{e}$ plane and is inclined at an angle $\Theta$ to the $z_{e}$ axis. The $y_{e}$-axis is identical with the $y_{c}$-axis. Then the $x_{c}-$ system is obtained from the $x_{e}$ - system by rotating the coordinate frame through an angle $\Theta$ with respect to the common y axis. 
The $x_{r}$ - system is obtained from the $x_{f}$ - system by rotating the coordinate frame through an angle $\tau_{R}$ with respect to the common $z$-axis, where (allowing for an arbitrary phase at $t=0$ )

$$
\tau_{R}=\omega_{R} t+\kappa_{R} \equiv 2 \pi v_{R} t+\kappa_{R}
$$

The $x_{t}$ - system is obtained from the $x_{e}$ - system by rotating the coordinate frame through an angle $\tau_{E}$ with respect to the common $z$-axis, where

$$
\tau_{E}=\omega_{E} t+\kappa_{E} \equiv 2 \pi v_{E} t+\kappa_{E}
$$

We are here approximating the motion of the Earth around the Sun to be purely circular motion with an angular velocity $\omega_{E}$ or a rotation frequency $v_{E}$.

The $x_{s}$ - system is obtained from the $x_{t}$ - system by rotating the coordinate frame through an angle $\pi / 2$ with respect to the common $y$-axis, so that $z_{s}$ is directed towards the Earth and the $x_{s}$ - axis is anti-parallel to the $z_{t}$-axis (i.e., the $z_{e}$-axis).

\section{THE DISTORTION FUNCTION}

We denote by $F(\theta, \phi, t)$ the "distortion function" of the rotator. In the case of real rotator, this distortion function might represent the magnetic field strength or departure from sphericity at the surface of the rotator; in the case of virtual rotator, it might represent, for instance, magnetic-field and/or velocity-field fluctuations of a traveling wave.

We express the distortion function in the form

$$
F(\theta, \phi, t)=\mathbf{F}(t) . \mathbf{Y}(\theta, \phi)
$$

where $\mathbf{Y}$ is the "vector" comprising the sequence of spherical harmonics, as defined in the appendix, that we truncate at order two: 


$$
\mathrm{Y}=\left(\mathrm{P}_{0}, \mathrm{P}_{1}, \mathrm{C}_{1}{ }^{1}, \mathrm{~S}_{1}{ }^{1}, \mathrm{P}_{2}, \mathrm{C}_{2}{ }^{1}, \mathrm{~S}_{2}{ }^{1}, \mathrm{C}_{2}{ }^{2}, \mathrm{~S}_{2}{ }^{2}\right),
$$

and the "vector" $F(t)$ is the sequence of coefficients

$$
F=\left(f_{0}, f_{1}, g_{1}{ }^{1}, h_{1}{ }^{1}, f_{2}, g_{2}{ }^{1}, h_{2}{ }^{1}, g_{2}{ }^{2}, h_{2}{ }^{2}\right),
$$

so that

$F(\theta, \phi, t)=f_{0} P_{0}+f_{1} P_{1}+g_{1}{ }^{1} C_{1}{ }^{1}+h_{1} 1 S_{1}{ }^{1+} f_{2} P_{2}+g_{2}{ }^{1} C_{2}{ }^{1}+h_{2}{ }^{1} S_{2}{ }^{1}+g_{2}{ }^{2} C_{2}{ }^{2}+h_{2}{ }^{2} S_{2}{ }^{2}$

In the $x_{r}$ frame that rotates with the rotator, the vector $F$ will be constant. We therefore write

$$
F_{\mathrm{r}}=\left(\mathrm{F}_{0}, \mathrm{~F}_{1}, \mathrm{G}_{1}{ }^{1}, \mathrm{H}_{1}{ }^{1}, \mathrm{~F}_{2}, \mathrm{G}_{2}{ }^{1}, \mathrm{H}_{2}{ }^{1}, \mathrm{G}_{2}{ }^{2}, \mathrm{H}_{2}{ }^{2}\right) \text {, }
$$

where $F_{0}$, etc., are independent of $t$. It will be convenient to write

$$
\begin{array}{ll}
\mathrm{G}_{1}{ }^{1}=\mathrm{L} \cos \kappa_{\mathrm{V}} & \mathrm{H}_{1}{ }^{1}=\mathrm{L} \sin \kappa_{\mathrm{L}} \\
\mathrm{G}_{2}{ }^{1}=\mathrm{M} \cos \kappa_{\mathrm{M}} & \mathrm{H}_{2}{ }^{1}=\mathrm{M} \sin \kappa_{\mathrm{M}} \\
\mathrm{G}_{2}{ }^{2}=\mathrm{N} \cos \kappa_{\mathrm{N}} & \mathrm{H}_{2}{ }^{2}=\mathrm{N} \sin \kappa_{\mathrm{N}}
\end{array}
$$

On noting that the $x_{f}$ - system is obtained from the $x_{r}$ - system by rotating the coordinate frame through an angle $\omega_{R} t$ with respect to the common $z$-axis, we may use equation (A.13), with $\Phi$ replaced by $-\omega_{R} t$, to express $F_{f}$ in terms of $F_{r}$. In this way we obtain the following form for $F_{f}$ :

$$
\begin{aligned}
& f_{0, f}=F_{0}, \\
& f_{1, f}=F_{1}, \\
& g_{1}{ }^{1}, f=L \cos \left(\tau_{R}+K_{L \prime}\right),
\end{aligned}
$$




$$
\begin{aligned}
& \mathrm{h}_{1}{ }^{1}{ }_{\mathrm{f}}=\mathrm{L} \sin \left(\tau_{\mathrm{R}}+\kappa_{\mathrm{L}}\right), \\
& f_{2, f}=F_{2} \text {, } \\
& \mathrm{g}_{2, \mathrm{f}}{ }^{1}=\mathrm{M} \cos \left(\tau_{\mathrm{R}}+\kappa_{\mathrm{M}}\right), \\
& h_{2}{ }^{1}, \mathrm{f}=M \sin \left(\tau_{R}+\kappa_{M}\right), \\
& \mathrm{g}^{2}{ }^{2}, \mathrm{f}=\mathrm{N} \cos \left(2 \tau_{\mathrm{R}}+\kappa_{\mathrm{N}}\right), \\
& h_{2}{ }^{2}, \mathrm{f}=N \sin \left(2 \tau_{\mathrm{R}}+\kappa_{N}\right) .
\end{aligned}
$$

On noting that the $x_{e}$-system is obtained from the $x_{f}$ - system by rotating the coordinate frame through an angle $-\Theta$ with respect to the common $y$-axis, we find from equation (A.16) of the Appendix that the components of the vector $F_{e}$ are given by

$$
\begin{aligned}
& \mathrm{f}_{0, \mathrm{e}}=\mathrm{F}_{0} \text { ， } \\
& f_{1, e}=\cos \Theta F_{1}-\sin \Theta L \cos \left(\tau_{R}+\kappa_{L}\right), \\
& g_{1}{ }^{1}, \mathrm{e}=\sin \Theta F_{1}+\cos \Theta L \cos \left(\tau_{R}+K_{L}\right), \\
& \mathrm{h}_{1}{ }^{1}, \mathrm{e}=\mathrm{L} \sin \left(\tau_{\mathrm{R}}+\mathrm{K}_{\mathrm{L}}\right), \\
& f_{2, e}=\frac{1}{2}\left(3 \cos ^{2} \theta-1\right) F_{2}-\frac{3}{2} \sin 2 \theta M \cos \left(\tau_{R}+\kappa_{M}\right)+3 \sin ^{2} \theta N \cos \left(2 \tau_{R}+\kappa_{N}\right), \\
& \mathrm{g}_{2}{ }^{1}, \mathrm{e}=\frac{1}{2} \sin 2 \theta \mathrm{F}_{2}+\cos 2 \theta \mathrm{M} \cos \left(\tau_{R}+\mathrm{K}_{\mathrm{M}}\right)-\sin 2 \theta \mathrm{N} \cos \left(2 \tau_{R}+\kappa_{N}\right) \\
& h_{2}{ }^{1}, \mathrm{e}=\cos \Theta M \sin \left(\tau_{R}+\kappa_{M}\right)-2 \sin \Theta N \sin \left(2 \tau_{R}+\kappa_{N}\right) \text {, } \\
& \mathrm{g}_{2}{ }^{2}, \mathrm{e}=\frac{1}{4} \sin ^{2} \Theta \mathrm{F}_{2}+\frac{1}{4} \sin 2 \Theta \mathrm{M} \cos \left(\tau_{\mathrm{R}}+\kappa_{\mathrm{M}}\right)+\frac{1}{2}\left(1+\cos ^{2} \Theta\right) \mathrm{N} \cos \left(2 \tau_{\mathrm{R}}+\kappa_{\mathrm{N}}\right),
\end{aligned}
$$




$$
h_{2}{ }^{2}, \mathrm{e}=\frac{1}{2} \sin \Theta M \sin \left(\tau_{R}+\kappa_{M}\right)+\cos \Theta N \sin \left(2 \tau_{R}+\kappa_{N}\right) .
$$

\section{THE ACCEPTANCE FUNCTION}

Solar activity comprises features and events that are distributed over the surface of the Sun. However, we frequently use indices to summarize the total activity observed at a certain time. For instance, the daily Zurich sunspot number is indicative of sunspot activity on the visible hemisphere.

In forming such indices, we are processing only data on the visible hemisphere. Furthermore, observations near the limb are likely to be degraded. In general, we may regard the "signal" (such as the sunspot number) as being formed from the activity distribution, that we denote by $f(\theta, \phi, t)$, by the equation

$$
S(t)=\frac{1}{4 \pi} \iint d \theta \sin \theta d \phi A(\theta, \phi) f(\theta, \phi),
$$

where the "acceptance function" $A(\theta, \phi)$ indicates the way in which different parts of the surface of the Sun contribute to the signal.

We may follow $\S 4$ in expressing the acceptance function in terms of spherical harmonics in the form

$$
\mathbf{A}(\theta, \phi, t)=\mathbf{A}(t) \cdot \mathbf{Y}(\theta, \phi),
$$

where the vector $Y$ is defined by equation (4.2) and we now write

$$
A=\left(u_{0}, u_{1}, v_{1}{ }^{1}, w_{1}{ }^{1}, u_{2}, v_{2}{ }^{1}, w_{2}{ }^{1}, v_{2}{ }^{2}, w_{2}^{2}\right)
$$

Once again, we retain only spherical harmonics up to the second order. Explicitly, 


$$
\begin{gathered}
\mathbf{A}(\theta, \phi, t)=u_{0} P_{0}+u_{1} P_{1}+v_{1}{ }^{1} C_{1}^{1}+w_{1}{ }^{1} S_{1}^{1} \\
+u_{2} P_{2}+v_{2}{ }^{1} C_{2}^{1}+w_{2}{ }^{1} S_{2}^{1}+v_{2}^{2} C_{2}^{2}+w_{2}^{2} S_{2}^{2}
\end{gathered}
$$

In terms of the $x_{\mathbf{s}}$ - coordinate system defined in $\S 3$, for which the $z$-axis always points towards the Earth, the acceptance function will clearly be cylindrically symmetrical, so that it is expressible as $A(\theta)$. In this system, the vector (5.3) simplifies to

$$
\mathbf{A}_{\mathrm{s}}=\left(\mathrm{U}_{0}, \mathrm{U}_{1}, 0,0, \mathrm{U}_{2}, 0,0,0,0\right) \text {, }
$$

where $U_{0}, U_{1}$ and $U_{2}$ are constants, defined in terms of $A(\theta)$ as follows:

$$
\begin{aligned}
& \mathrm{U}_{0}=\frac{1}{2} \int_{0}^{\pi} \mathrm{d} \theta \sin \theta \mathrm{A}(\theta), \\
& \mathrm{U}_{1}=\frac{3}{2} \int_{0}^{\pi} \mathrm{d} \theta \sin \theta \cos \theta \mathrm{A}(\theta), \\
& \mathrm{U}_{2}=\frac{5}{4} \int_{0}^{\pi} \mathrm{d} \theta \sin \theta\left(3 \cos ^{2} \theta-1\right) \mathrm{A}(\theta) .
\end{aligned}
$$

For the special case that

$$
\left.\begin{array}{l}
A(\theta)=1, \quad 0<\theta<\theta_{a} \\
A(\theta)=0, \quad \theta>\theta_{a},
\end{array}\right\}
$$

we find that

$$
\begin{aligned}
\mathrm{U}_{0} & =\sin ^{2} \frac{1}{2} \theta_{\mathrm{a}}, \\
\mathrm{U}_{1} & =\frac{3}{4} \sin ^{2} \theta_{\mathrm{a}}, \\
\mathrm{U}_{2} & =\frac{5}{4} \sin ^{2} \theta_{\mathrm{a}} \cos \theta_{\mathrm{a}} .
\end{aligned}
$$


It is interesting to note that

$$
\text { If } \theta_{\mathrm{a}}=\pi / 2, \mathrm{U}_{0}=1 / 2, \mathrm{U}_{1}=3 / 4, \mathrm{U}_{2}=0
$$

That is to say, if an index were compiled from perfect observations over the visible hemisphere, we would be "blind" to spherical harmonics of second order.

We now consider the transformation from the $\mathbf{x}_{\mathbf{s}}$ - coordinate system to the $\mathbf{x}_{\mathbf{t}}$ -coordinate system. The $x_{t}$ - system is formed from the $x_{\mathbf{s}}$ - system by a rotation by the angle $-\pi / 2$ about the $y$ axis. On using equation (A.16), we find that

$$
\begin{gathered}
\mathrm{u}_{0, \mathrm{t}}=\mathrm{U}_{0}, \quad \mathrm{u}_{1, \mathrm{t}}=0, \quad \mathrm{v}_{1, \mathrm{t}}^{1}=\mathrm{U}_{1}, \quad \mathrm{w}_{1, \mathrm{t}}^{1}=0, \\
\mathrm{u}_{2, \mathrm{t}}=-\frac{1}{2} \mathrm{U}_{2}, \quad \mathrm{v}_{2, \mathrm{t}}^{1}=0, \quad \mathbf{w}_{2, \mathrm{t}}^{1}=0, \quad \mathrm{v}_{2, \mathrm{t}}^{2}=\frac{1}{4} U_{2}, \quad \mathbf{w}_{2, \mathrm{t}}^{2}=0 .
\end{gathered}
$$

\section{THE SIGNAL}

As explained in $\S 1$, we are in this article examining only the interaction between a hypothetical rotator and an envelope that is assumed to be spherically symmetric. If $F(\theta, \phi, t)$ is now regarded as the disturbance in surface activity due to the rotator, we may assume that this function is stationary in a frame that rotates with the rotator. In this way, we are led to examine the properties of a "signal" that is formed from the distortion function and the acceptance function as follows:

$$
S(t)=\iint d \theta \sin \theta d \phi A(\theta, \phi, t) F(\theta, \phi, t) .
$$

On expressing $F(\theta, \phi, t)$ as in equation (3.4) and $A(\theta, \phi, t)$ as in equation (5.4), we find that $S(t)$ may be expressed as

$$
S=u_{0} f_{0}+\frac{1}{3} u_{1} f_{1}+\frac{1}{3} v_{1}^{1} g_{1}^{1}+\frac{1}{3} w_{1}^{1} h_{1}^{1}
$$




$$
+\frac{1}{5} \mathrm{u}_{2} \mathrm{f}_{2}+\frac{3}{5} \mathrm{v}_{2}{ }^{1} \mathrm{~g}_{2}{ }^{1}+\frac{3}{5} \mathrm{w}_{2}{ }^{1} \mathrm{~h}_{2}{ }^{1}+\frac{12}{5} \mathrm{v}_{2}{ }^{2} \mathrm{~g}_{2}{ }^{2}+\frac{12}{5} \mathrm{w}_{2}{ }^{2} \mathrm{~h}_{2}{ }^{2}
$$

We may carry out this evaluation in any coordinate system. It is most convenient to use the $x_{e}$-system. In terms of this system the quantities $f_{0}$, etc., are given by equation (4.7). It is therefore necessary to transform the acceptance function into the same frame.

We see from $\S 3$ that the $x_{e}$ - system is obtained from the $x_{t}$ - system by a rotation by the angle $-\tau_{\mathrm{E}}$ about the common $\mathrm{z}$-axis, where $\tau_{\mathrm{E}}$ is given by equation (3.2). Hence, on using equation (A.13), we find that

$$
\begin{gathered}
u_{0, \mathrm{e}}=U_{0}, u_{1, \mathrm{e}}=0, v_{1, \mathrm{e}}^{1}=U_{1} \cos \tau_{\mathrm{E}}, \mathrm{w}_{1, \mathrm{e}}^{1}=U_{1} \sin \tau_{\mathrm{E}} \\
\mathrm{u}_{2, \mathrm{e}}=-\frac{1}{2} U_{2}, v_{2, \mathrm{e}}^{1}=0, \mathrm{w}_{2, \mathrm{e}}^{1}=0, \mathrm{v}_{2, \mathrm{e}}^{2}=\frac{1}{4} U_{2} \cos 2 \tau_{\mathrm{E}}, \mathrm{w}_{2, \mathrm{e}}^{2}=\frac{1}{4} U_{2} \sin 2 \tau_{\mathrm{E}}
\end{gathered}
$$

On combining equations (4.8), (6.2) and (6.3), and noting equation (3.1) and (3.2), we obtain the following expression for the signal:

$$
\begin{aligned}
S & =U_{0} F_{0}-\frac{1}{20}\left(3 \cos ^{2} \Theta-1\right) U_{2} F_{2} \\
& +\frac{1}{3} U_{1} F_{1} \sin \Theta \cos \left(\omega_{E} t+\kappa_{E}\right)+\frac{3}{20} U_{2} F_{2} \sin ^{2} \Theta \cos \left(2 \omega_{E} t+2 \kappa_{E}\right) \\
& +\frac{3}{20} U_{2} M \sin 2 \Theta \cos \left(\omega_{R} t+\kappa_{R}+\kappa_{M}\right) \\
& -\frac{1}{6} U_{1} L(1-\cos \Theta) \cos \left(\left(\omega_{R}+\omega_{E}\right) t+\kappa_{R}+\kappa_{E}+\kappa_{L}\right) \\
& +\frac{1}{6} U_{1} L(1+\cos \Theta) \cos \left(\left(\omega_{R}-\omega_{E}\right) t+\kappa_{R}-\kappa_{E}+\kappa_{L}\right) \\
& -\frac{3}{20} U_{2} M \sin \Theta(1-\cos \theta) \cos \left(\left(\omega_{R}+2 \omega_{E}\right) t+\kappa_{R}+2 \kappa_{E}+\kappa_{M}\right) \\
& +\frac{3}{20} U_{2} M \sin \Theta(1+\cos \Theta) \cos \left(\left(\omega_{R}-2 \omega_{E}\right) t+\kappa_{R}-2 \kappa_{E}+\kappa_{M}\right) \\
& -\frac{3}{10} U_{2} N \sin ^{2} \Theta \cos \left(2 \omega_{R} t+2 \kappa_{R}+\kappa_{N}\right)
\end{aligned}
$$




$$
\begin{aligned}
& +\frac{3}{20} U_{2} N(1-\cos \Theta)^{2} \cos \left(\left(2 \omega_{R}+2 \omega_{E}\right) t+2 \kappa_{R}+2 \kappa_{E}+\kappa_{N}\right) \\
& +\frac{3}{20} U_{2} N(1+\cos \theta)^{2} \cos \left(\left(2 \omega_{R}-2 \omega_{E}\right) t+2 \kappa_{R}-2 \kappa_{E}+\kappa_{N}\right)
\end{aligned}
$$

\section{DISCUSSION}

We now interpret the results of $\S 2$ in the light of the analysis of $\S \S 3-6$.

We first note that our proposal of an oblique rotator (or oscillator) with a frequency of $447.5 \mathrm{nHz}$ (period 25.86 days) offers an explanation of nine periodicities in solar activity: four periodicities that are shown in Figures 1 and 2, and five periodicities, multiples of 25.86 days, that comprise the "154-day" complex of periodicities.

Neither the Zurich nor the Greenwich spectrum shows significant evidence for any sidebands of the fundamental. On referring to (6.4), we see that this absence is understandable if both $\mathrm{L}$ and $\mathrm{M}$ are small. In analyzing the same spectra, we found good evidence for the harmonic triplet in the Greenwich spectrum, and strong evidence for one of these three peaks in the Zurich spectrum. This implies that $N$ differs significantly from zero. The fact that the three peaks of the triplet in the Greenwich spectrum have comparable magnitudes suggests that $\Theta$ is not small (and is not near to $\pi$ either). The fact that the triplet is stronger in the Greenwich spectrum than in the Zurich spectrum suggests that $U_{2}$ is larger for the Greenwich data compilation than for the Zurich data compilation. It will be possible to evaluate this conjecture when we complete our analysis (now in hand) of the sunspot position data in the Greenwich compilation.

However, we also need to understand the fact that the Zurich spectrum shows a strong peak at the fundamental. This cannot be attributed to our present model, 
since we infer from the absence of sidebands of the fundamental that $M$ is small. A more realistic model would take account of the fact that sunspots are not formed uniformly over the envelope of the Sun, but only in certain latitude bands known as the "sunspot zones." We plan to examine this more complex model to see if it offers an interpretation of the presence of a strong fundamental and the apparent absence of sidebands of the fundamental.

It is notable that the periodicity determined by analysis of sunspot data appears to have a very narrow bandwidth. This is to be expected of a real rotator. However, as two referees have pointed out in some detail, there are severe theoretical problems to be faced in considering that, for instance, the radiative zone contains a "core" that rotates about an axis different from that of the radiative zone itself. Mestel and Weiss (1987) showed that, even if the magnetic axis is aligned with the rotation axis, there is unlikely to be any significant departure from isorotation in the Sun's radiative zone if that zone is permeated by a magnetic field of strength exceeding $.03 \mathrm{G}$, that they consider to unrealistically low. In principle, differential rotation in the radiative zone could lead to magnetic decoupling of two or more regions, but this would in turn lead to high gradients of the angular velocity at the boundary layers, and these gradients would be expected to lead to shear instabilities (Zahn 1983) that would act in such a way as to restore isorotation. Moss, Mestel and Tayler (1990) have shown that, if the magnetic axis is not aligned with the rotation axis, the radiative region of a star should settle into a state of isorotation on a time scale that is quite short compared with its lifetime.

On the other hand, if the signal is due to a "virtual rotator" associated with a traveling-wave disturbance, one might be surprised that the frequency of the "clock" is so sharply defined. However, a traveling-wave disturbance is likely to be long-lived only if it is maintained by an active mechanism - for instance, if it is the result of an 
instability. In that case, depending on the nature of the instability, the resulting frequency could be sharply defined.

We can readily identify three programs of data analysis that should be undertaken to further evaluate our proposal that the Sun contains a clock that regulates the 154-day complex of periodicities:-

(i) One should analyze sunspot position data in the Greenwich data set rather than work only with sunspot area data. This should provide a more stringent test of our hypothesis. Furthermore, if there really is an oblique rotator (real or virtual), such an analysis should provide evidence concerning the direction of the axis of that rotator. This work is in hand.

(ii) Since the 154-day and related periodicities show up very strongly in solar activity data, one should analyze flare data to search for evidence of an oblique rotator. This work also is in hand.

(iii) One should re-analyze available helioseismology data to examine the possibility that some internal region of the Sun may have a rotation axis that is very different from that of the Sun's surface.

Earlier drafts of this article were circulated in the solar physics community during 1991. We have learned through private communications that, in response to this proposal, Philip Goode of the New Jersey Institute of Technology and Mike Thomson of Cambridge University have kindly undertaken such an analysis and concluded that it is possible to rule out a real oblique rotator with a radius of $0.7 R_{O}$ that is characterized by a single angular velocity vector. We have also learned through private communications that Douglas Gough of Cambridge University and Alexander Kosovichev of the Crimean Astrophysical Observatory have undertaken a similar analysis and concluded that it is not possible to rule out an oblique real rotator if the rotation rate is allowed to be a free function of radius. However, when the rotation rate is taken to be the value $v_{R}$ that is determined from our spectrum 
analysis, Gough and Kosovichev also rule out the possibility that the entire radiative zone is rotating at an oblique angle with respect to the Sun's visible envelope.

We wish to thank Richard Bogart, Douglas Gough, Alexander Kosovichev, John Leibacher, Eugene Parker, Phillip Scherrer and William Thompson for their advice on special points that arose in the course of this study, and to express our gratitude to Philip Goode, Douglas Gough, Alexander Kosovichev and Mike Thomson for advising us of the results of their investigations in advance of publication. We also thank two anonymous referees for their valuable comments and suggestions, which have we believe led to considerable improvement in this article. This work was supported in part by the Air Force grant F49620-92-J-0015, NASA grant NAGW-2265, ONR grant N00014-85-K-011, and Lockheed Solar-A subcontract NAS8-37334. 


\section{APPENDIX: SPHERICAL HARMONICS}

We follow the notation of Whittaker and Watson (1952). Carrying our analysis only to second order, we note that the Legendre polynomials are given by

$$
\begin{aligned}
& P_{0}=1 \\
& P_{1}=\mu \\
& P_{2}=\frac{1}{2}\left(3 \mu^{2}-1\right)
\end{aligned}
$$

where we write $\mu$ for $\cos \theta$. The relevant associated Legendre polynomials are given by

$$
\left.\begin{array}{l}
P_{1}{ }^{1}=\left(1-\mu^{2}\right)^{1 / 2}, \\
P_{2}^{1}=3 \mu\left(1-\mu^{2}\right)^{1 / 2} \\
P_{2}{ }^{2}=3\left(1-\mu^{2}\right)
\end{array}\right\}
$$

Following Chapman and Bartels (1940), we use the following notation for spherical harmonics:

$$
\left.\begin{array}{l}
C_{1}^{m}=R^{m}(\cos \theta) \cos m \phi, \\
S^{m}=P_{1}^{m}(\cos \theta) \sin m \phi .
\end{array}\right\}
$$

Any function $F(\theta, \phi)$ may be expressed, up to second order, in the form

$$
F(\theta, \phi)=F_{r} Y_{r}
$$

where the "vector" $\mathbf{Y}$ is the sequence of spherical harmonics,

$$
Y=\left(P_{0}, P_{1}, C_{1}{ }^{1}, S_{1}{ }^{1}, P_{2}, C_{2}{ }^{1}, S_{2}{ }^{1}, C_{2}{ }^{2}, S_{2}{ }^{2}\right),
$$

and $F$ is the relevant set of coefficients that we write in the form 


$$
F=\left(f_{0}, f_{1}, g_{1}{ }^{1}, h_{1}{ }^{1}, f_{2}, g_{2}{ }^{1}, h_{2}{ }^{1}, g_{2}{ }^{2}, h_{2}{ }^{2}\right)
$$

It is convenient to express the spherical harmonics in terms of the components of the unit vector, related to the polar angles $(\theta, \phi)$ by

$$
\begin{aligned}
& l_{x}=\sin \theta \cos \phi \\
& l_{y}=\sin \theta \sin \phi, \\
& l_{z}=\cos \theta
\end{aligned}
$$

We find that

$$
\begin{aligned}
P_{0}=1, P_{1}=l_{z}, C_{1}{ }^{1}= & l_{x}, S_{1}{ }^{1}=l_{y}, P_{2}=(1 / 2)\left(3 l_{z}^{2}-1\right), \\
& C_{2}{ }^{1}=3 l_{z} l_{x}, S_{2}{ }^{1}=3 l_{z} l_{y}, C_{2}^{2}=3\left(l_{x}^{2}-l_{y}^{2}\right), S_{2}^{2}=6 l_{x} l_{y} .
\end{aligned}
$$

We now consider a second set of axes, and write the components of the vector as $\left(\hat{l}_{\mathbf{x}}, \hat{l}_{y}, \hat{l}_{z}\right)$, etc. If the spherical harmonics are related by

$$
\widehat{Y}_{\mathrm{r}}=\mathrm{M}_{\mathrm{rs}} \mathrm{Y}_{\mathrm{s}} \text {, }
$$

we find that the coefficients are related by

$$
\widehat{F}_{\mathrm{r}}=\left(\mathrm{M}^{-1}\right)_{\mathrm{sr}} \mathrm{F}_{\mathrm{s}} \text {. }
$$

If the coordinate system $\hat{\mathbf{x}}$ is formed from the system $\mathbf{x}$ by rotation by the angle $\Phi$ about the z-axis, so that

$$
\left[\begin{array}{l}
\hat{l}_{x} \\
\hat{l}_{y} \\
\hat{l}_{z}
\end{array}\right]=\left[\begin{array}{ccc}
\cos \Phi & \sin \Phi & \cdot \\
-\sin \Phi & \cos \Phi & \cdot \\
\cdot & \cdot & 1
\end{array}\right]\left[\begin{array}{l}
l_{x} \\
l_{y} \\
l_{z}
\end{array}\right]
$$

we find that the spherical-harmonic vectors are related by 


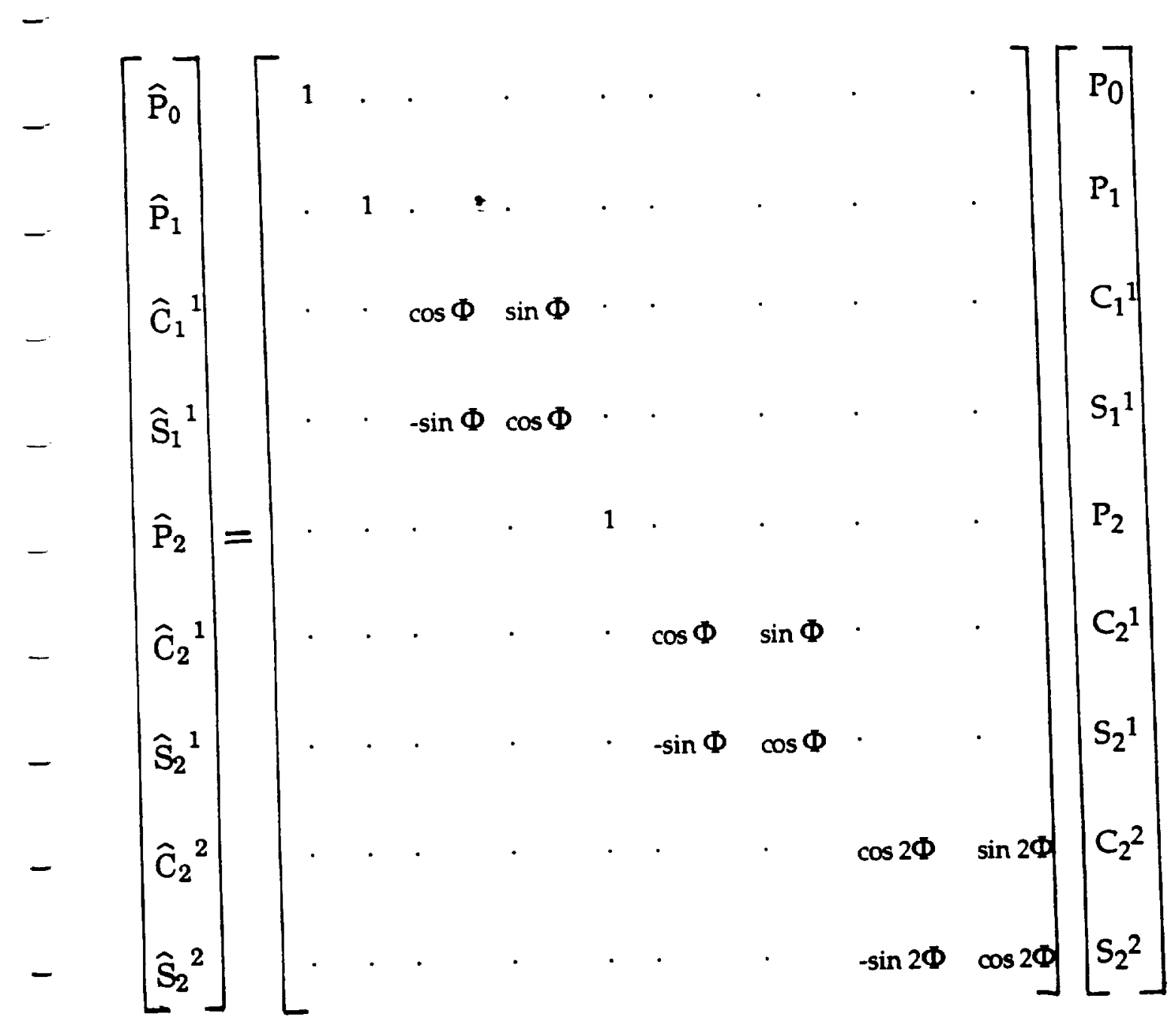

(A.12)

- The inverse matrix is found by replacing $\Phi$ by $-\Phi$. Hence we find that the two sets of coefficients of the spherical harmonics are related by 


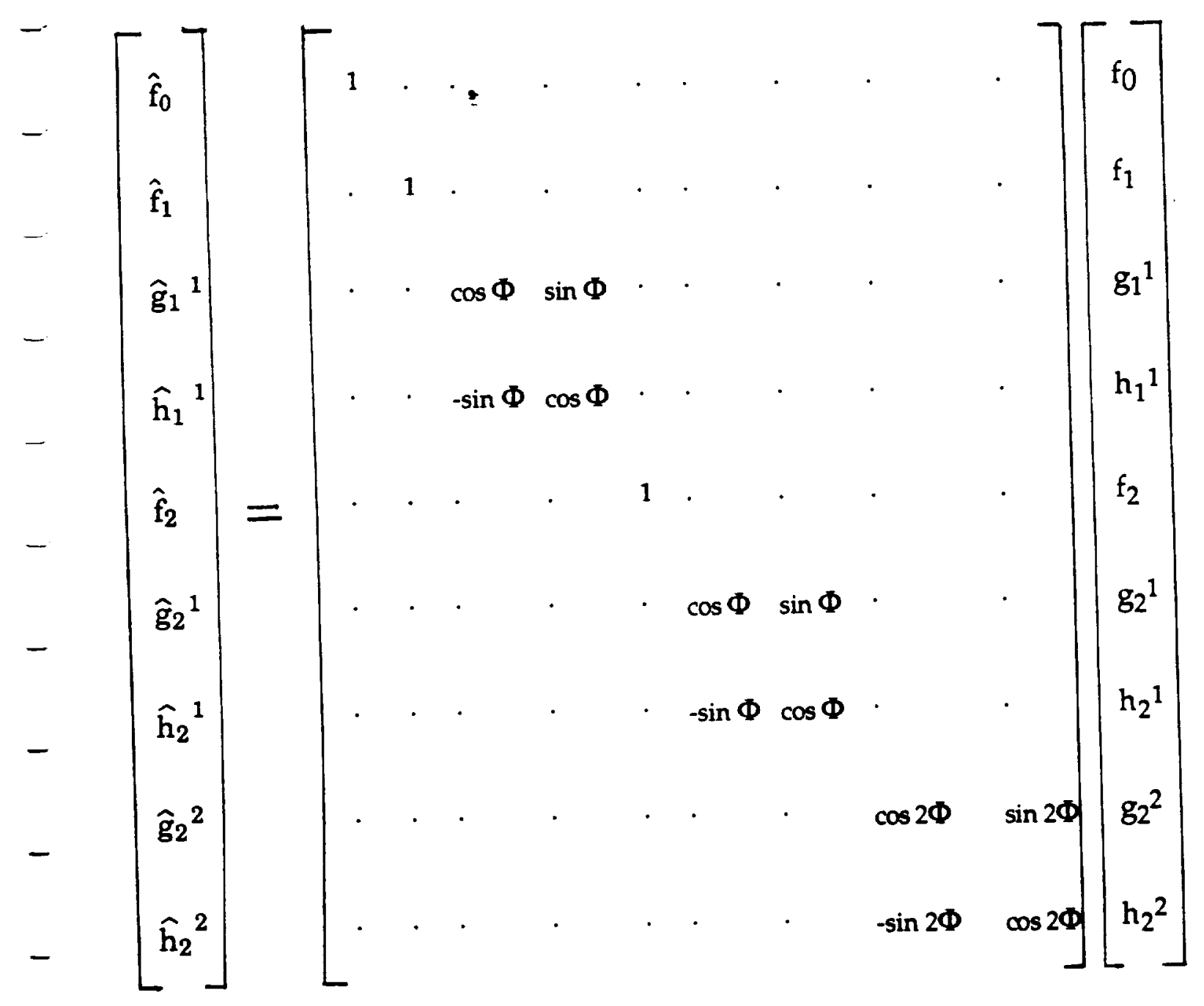

(A.13)

Similarly, we may consider the transformation of forming the new coordinate system $\hat{\mathbf{x}}$ from the coordinate system $\mathbf{x}$ by rotation by an angle $\Theta$ about the $y$-axis. The unit vectors are now related by 


$$
=\left[\begin{array}{l}
\hat{i}_{x} \\
\hat{r}_{y} \\
\hat{i}_{z}
\end{array}\right]=\left[\begin{array}{ccc}
\cos \Theta & \cdot & -\sin \Theta \\
\cdot & 1 & \cdot \\
\sin \Theta & \cdot & \cos \Theta
\end{array}\right]\left[\begin{array}{l}
l_{x} \\
l_{y} \\
l_{z}
\end{array}\right]
$$

Hence, we find that the spherical-harmonic vectors are related by
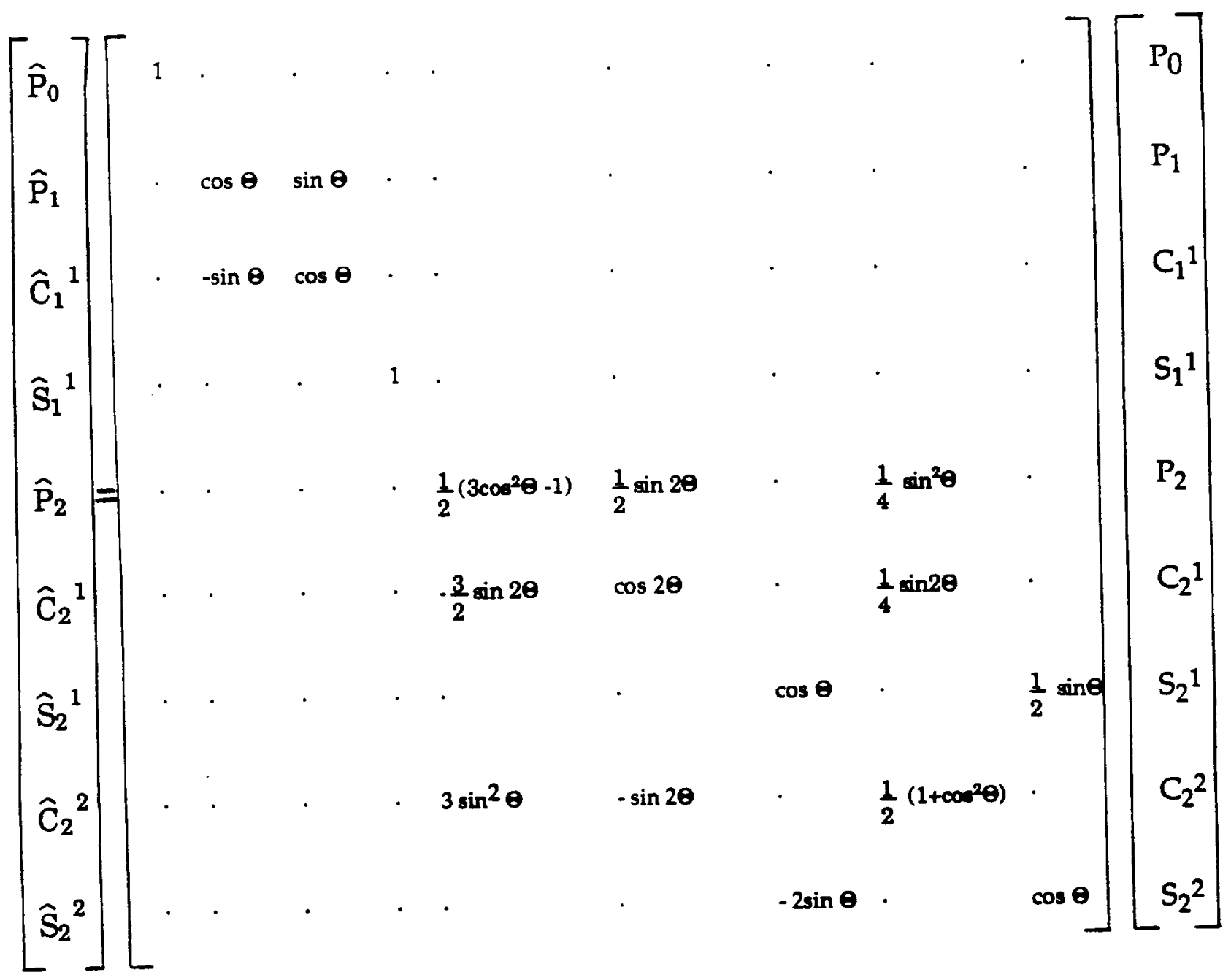

(A.15)

The inverse matrix is found by replacing $\Theta$ by $-\theta$. In this way, we find that the two sets of coefficients are related by 


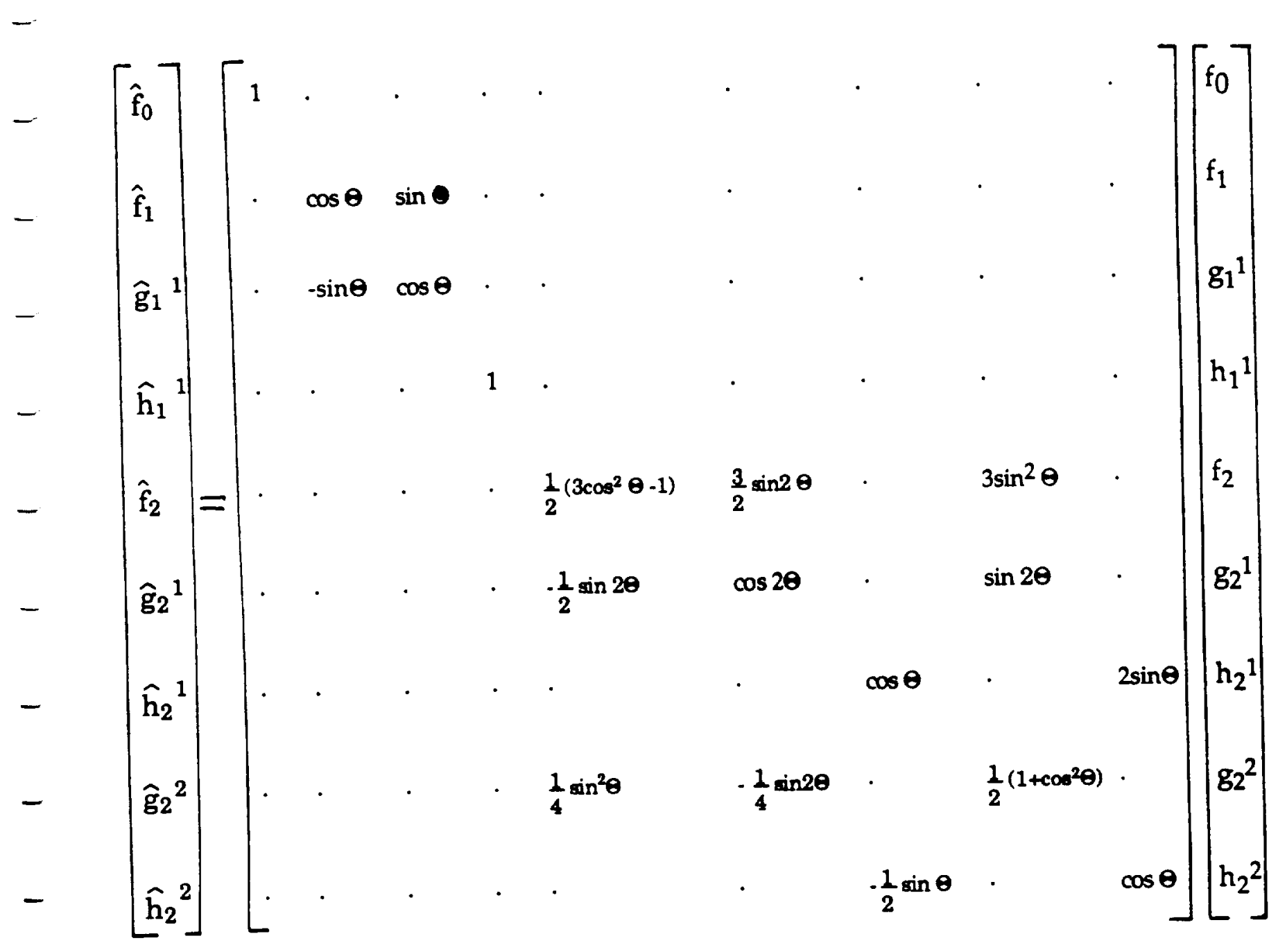

(A.16) 


\section{REFERENCES}

Andrews, D. G., Holton, J. R., and Leovy, C. B. 1987, Middle Atmosphere Dynamics (Orland: Academic Press)., Chapter 8.

Bai, T. 1987, Ap. J., 314, 795.

Bai, T., and Cliver, E. W. 1990, Ap. J. 363299.

Bai, T., and Sturrock, P. A. 1987, Nature, 327, 601.

Bai, T., and Sturrock, P. A. 1991, Nature, 350, 141.

Bogart , R.S., and Bai, T. 1985, Ap. J. (Letters), 299, L51.

Chapman, S., and Bartels, J. 1940, Geomagnetism (Oxford: Clarendon Press), pp. 606 et seq.

Dicke, R.H. 1976, Solar Phys., 47, 475.

Dodson H.W., and Hedeman, E.R. 1971, An Experimental Comprehensive Flare Index and its Derivation for "Major" Flares, 1955-1969. World Data Center for Solar-Terrestrial Physics Report UAG-14 (Boulder: NOAA).

Dodson H.W., and Hedeman, E.R. 1976, Experimental Comprehensive Solar Flare Indices for Certain Flares, 1970-1974, World Data Center for SolarTerrestrial Physics Report UAG-62 (Boulder: NOAA).

Dodson H.W., and Hedeman, E.R. 1981, Experimental Comprehensive Solar Flare Indices for "Major" and Certain Lesser Flares, 1975-1979, World Data Center for Solar-Terrestrial Physics Report UAG-80 (Boulder: NOAA).

Hale, G. E. 1924 Nature 113, 105.

Haubold, H. J., and Gerth, E. 1990, Solar Phys. 127, 347.

Ichimoto, K., Kubota, J., Suzuki, M., Tohmura, I., and Kurokawa, H. 1985, Nature, 316, 422.

Knight, J.W., Schatten, K.H., and Sturrock, P.A. 1979, Ap. J. (Letters), 227, L153.

Mestel, L., and Weiss, N.O. 1987, M.N.R.A.S., 226, 123.

Moss, D.L., Mestel, L., and Tayler, R.J. 1990, M.N.R.A.S., 245, 550. 
Ribes, E., Merlin, Ph., Ribes, J.-C., and Barthalot, R. 1989, Annales Geophys. 89$04,321$.

Rieger, E., Share, G. H., Forrest, D. J., Kanbach, G., Reppin, C., and Chupp, E. L. 1984, Nature, 312, 623.

Sakurai, K. 1979, Nature 278, 146.

Scherrer, P. S. 1983, in Solar Seismology from Space. A conference at Snowmass, Colorado, August 17-19, 1983 (eds. R.K. Ulrich, J. Harvey, E.J.Rhodes Jr., J. Toomre. JPL Publ. 84-84, NASA Jet Propulsion Laboratory, Pasadena, California) pp. 173-182.

Schwabe, H. (1844) Astron. Nachr. 21, 233.

Whittaker, E.T., and Watson, G.N. 1952, A Course of Modern Analysis (Cambridge University Press), pp. 393 et seq.

Wolff, C.L., 1983, Ap.J., 264, 667.

Zahn, J.-P. 1983, Astrophysical Processes in Upper-Main-Sequence Stars (eds. B. Hauck and A. Maeder; Sauvery, Switzerland, Geneva Observatory), p. 253. 


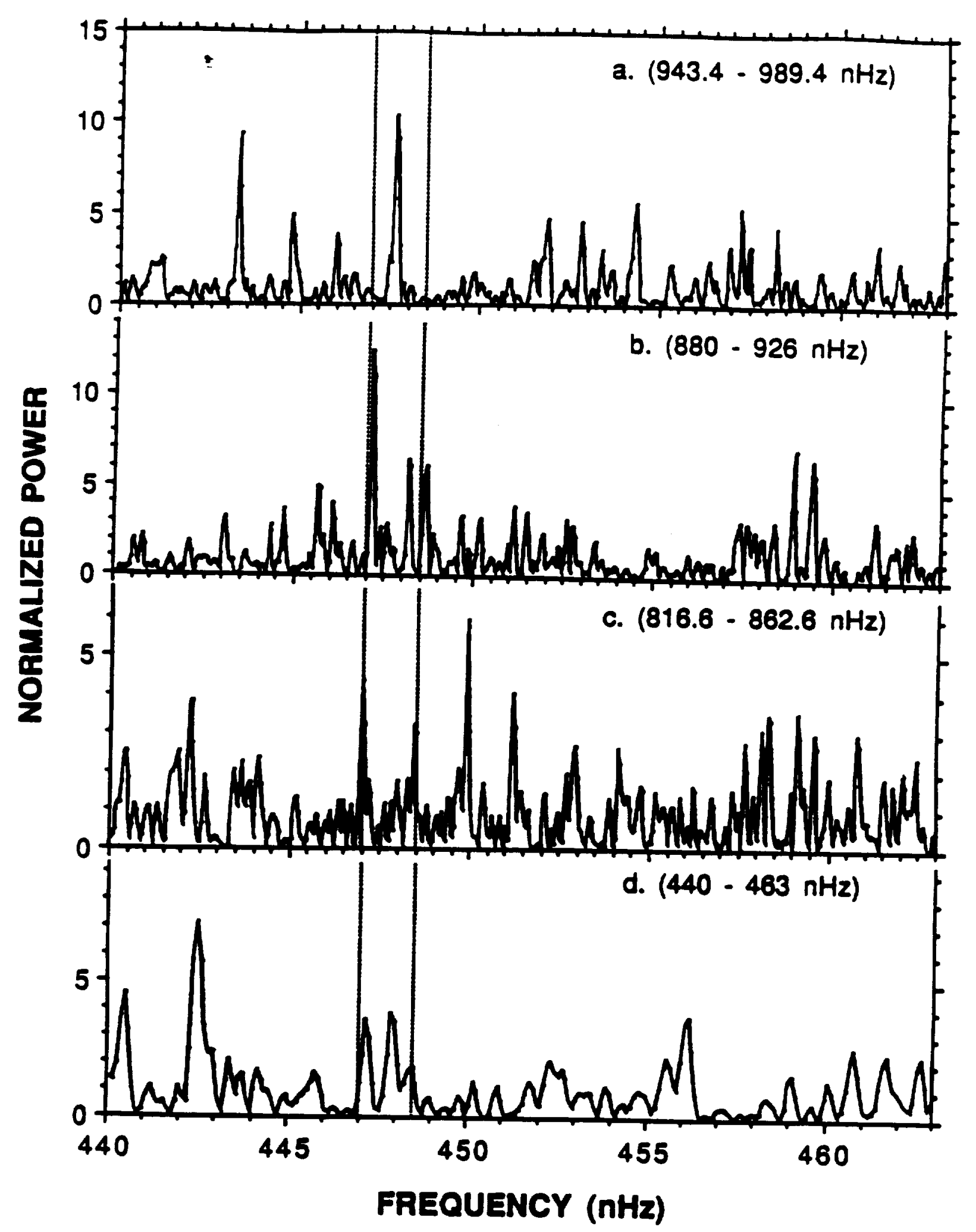

Figure 2 